\title{
The Interaction Between Recombinant Protein F Derived from Nontypeable Haemophilus influenzae and Lipoprotein(a)
}

\author{
Liu En ${ }^{1,2}$, Li Weng-long ${ }^{1,2}$, Han Run-lin ${ }^{1,2, *}$ \\ ${ }^{1}$ College of Veterinary Science, Inner Mongolia Agriculture University, Hohhot, China \\ ${ }^{2}$ Research Center of Plasma Lipoprotein Immunology, Inner Mongolia Agriculture University, Hohhot, China
}

Eamil address:

han-runlin@163.com (Han Run-lin), liuen1989xiaoxiang@163.com (Liu En)

\section{To cite this article:}

Liu En, Li Weng-long, Han Run-lin. The Interaction Between Recombinant Protein F Derived from Nontypeable Haemophilus influenzae and Lipoprotein(a). American Journal of Clinical and Experimental Medicine. Vol. 3, No. 6, 2015, pp. 338-343.

doi: 10.11648/j.ajcem.20150306.11

\begin{abstract}
Protein F (PF) is a surface plasminogen (Plg) receptor on the nontypeable Haemophilus influenzae (NTHi). Plg via its lysine binding sites (LBS) can bind to PF. Apolipoprotein(a) [Apo(a)] is one component of Lipoprotein(a) [Lp(a)]. It has Kringle (K) domains, which contain LBS and has a high homology with Plg. Therefore, we speculated that Lp(a) might bind to Plg receptor on the surface of NTHi, subsequently competitively inhibiting the interaction of NTHi with Plg. In this study, recombinant $\mathrm{PF}(\mathrm{rPF})$ and its $\mathrm{C}$-terminal lysine residue-deleted variant (rPF $\triangle \mathrm{K})$ were expressed in E. coli BL21. The interactions of rPF with Plg and Lp(a) were tested by ELISA. The results showed that rPF could bind to Plg and Lp(a). The binding capacity of rPF was significantly higher than that of rPF $\Delta \mathrm{K}$. The interactions of rPF with Plg and Lp(a) could be inhibited by EACA. $2 \mathrm{mmol} / \mathrm{L}$ of EACA significantly inhibited the binding of rPF to Plg, while $0.2 \mathrm{mmol} / \mathrm{L}$ of EACA could significantly reduce the binding of $\mathrm{rPF}$ to $\mathrm{Lp}(\mathrm{a}) .50 \mathrm{ng} / 100 \mu \mathrm{L} \mathrm{Lp}$ (a) could significantly inhibit the interaction of $\mathrm{rPF}$ with Plg. In addition, affinity chromatography assay followed by Western biotting was also used to study the interaction. In overall, C-terminal lysine residue of rPF and the lysine binding sites (LBS) of Plg and Lp(a) should be responsible for these specifically bindings. Lp(a) could combine with rPF consequently inhibiting the interaction of Plg with rPF. This revealed that $\mathrm{Lp}$ (a) might play a role in anti-NTHi infection.
\end{abstract}

Keywords: Nontypeable Haemophilus Influenzae, Plasminogen, Lipoprotein(a), Recombinant Protein F

\section{重组不可分型流感嗜血杆菌F蛋白与脂蛋白（a）的相互作用}

刘恩 ${ }^{1,2}$, 李文龙 ${ }^{1,2}$, 韩润林 ${ }^{1,2, *}$

${ }^{1}$ 兽医学院, 内蒙古农业大学, 呼和浩特, 中国

${ }^{2}$ 血浆脂蛋白免疫学研究中心, 内蒙古农业大学, 呼和浩特, 中国

邮箱

han-runlin@163. com（韩润林），1iuen1989xiaoxiang@163.com（刘恩）

\begin{abstract}
摘要: F蛋白 (Protein F, PF) 是不可分型流感嗜血杆菌（nontypeable Haemophilus influenzae, NTHi) 表面的一种 纤溶酶原 (Plg) 受体。Plg利用自身的赖氨酸结合位点 (LBS) 与PF结合。脂蛋白 (a) [Lipoprotein (a), Lp (a)]中的载 脂蛋白 [Apolipoprotein (a), Apo (a)] 与 Plg 都具有K结构域, 两者高度同源。基于此, 本实验研究了 PF与 $\mathrm{P} 1 \mathrm{~g}$ 、 $\mathrm{Lp}(\mathrm{a})$ 的相互作用, 并推测Lp (a) 通过与NTHi上的P $1 g$ 受体结合, 竞争性抑制NTHi与P1g的结合。本实验利用大肠杆菌BL21表达 得到重组PF ( $r P F)$ 及缺失末端赖氨酸残基的PF $\Delta \mathrm{k}(r \mathrm{PF} \Delta \mathrm{k})$ 。通过ELISA试验检测 $r P F$ 与 $P 1 g 、 L p(a)$ 的相互作用, 结 果显示：rPF能够与 Plg、 $L p(a)$ 结合，且 $r P F$ 组与 Plg、 $L p(a)$ 的结合显著高于rPF $\Delta k$ 组。6-氨基已酸（EACA）能够抑制 $r P F$ 与 P lg、 Lp (a) 的结合, 当浓度达到 $2 \mathrm{mmol} / \mathrm{L}$ 时EACA对 $r \mathrm{PF}$ 与Plg结合的抑制作用显著增强。对 $r P F$ 与 $L p$ (a) 结合的抑制 在浓度为 $0.2 \mathrm{mmol} / \mathrm{L}$ 时明显增强。 $\mathrm{Lp}(\mathrm{a})$ 浓度达到 $50 \mathrm{ng} / 100 \mu \mathrm{L}$ 时, 能够显著的抑制 $\mathrm{rPF}$ 与 $\mathrm{P} 1 \mathrm{~g}$ 结合。此外, 亲和色谱 层析和Western blot也证明 rPF与 P $1 g$ 、 Lp (a) 的特异性结合主要是通过P $1 g$ 、 Lp (a) 的LBS与 $r P F$ 羧基末端的赖氨酸残基作 用进行的。Lp (a) 对 rPF与Plg结合的竞争性抑制作用表明Lp (a) 在抗NTHi感染过程中可能具有重要作用。
\end{abstract}

关键词：不可分型流感嗜血杆菌, 纤溶酶原, 脂蛋白（a），重组F蛋白 


\section{1. 引言}

流感嗜血杆菌（Haemophilus influenzae, Hi) 发 现于1892年，是一种革兰阴性球杆菌，可以引起不同的 疾病。根据荚膜多糖的有无可以分为荚膜型（可分型） 及非荚膜型（不可分型）。荚膜是侵袭性Hi主要的毒力 因子, 荚膜型菌株又分为六个型 $(a-f)$, 其中b型 (Hib) 毒力最强 [1]。二十世纪九十年代Hib结合疫苗的应用使 得由Hib引起的侵袭性疾病的发病率显著降低 [2]。和荚 膜型 Hi 不同，不可分型流感嗜血杆菌（nontypeable Haemophilus influenzae, NTHi) 是人体特别是儿童呼 吸道内的共栖菌, 很少引起侵袭性疾病 [3]。NTHi也是一 种条件致病菌, 当宿主免疫机能降低时能够造成感染。 NTHi 主要引起呼吸道局限性疾病如急性中耳炎、支气管 炎、鼻窦炎等 $[4-5]$ 。和许多病原菌一样, NTHi 能够利用 $\mathrm{P} 1 \mathrm{~g}$ 秥附到宿主的胞外基质（ECM）对其进行破坏，进而 对宿主造成损伤。

P $1 g$ 是一种单链糖蛋白, 是人体纤溶系统的重要组成 成分。P 1 由 由 791 个氨基酸构成, 分子量大约为 $90 \mathrm{kD}$, 具 有 7 个结构域 [6]。构成P $1 \mathrm{~g}$ 的 7 个结构域中有 5 个 Kringle 结构域 (K1, K2, K3, K4, K5), 这5个结构域中都包含LBS, 此位点可识别 $P 1 g$ 受体羧基末端的赖氨酸残基, 而赖氨酸 或赖氨酸类似物 (如EACA, 氨甲环酸等) 可竞争性抑制P $1 \mathrm{~g}$ 与其受体的结合 [7]。包括铜绿假单胞菌, 伯氏疏螺旋体, 肺炎链球菌, 金黄色葡萄球菌和白色念珠菌在内的大多数 病原微生物都能同时表达几种不同的P $1 \mathrm{~g}$ 受体。已有的研 究发现, NTHi 表面的E蛋白 [8]、天冬氨酸酶 [9] 及新发现 的P4蛋白 [10]都是NTHi上的P1g受体。PF是NTHi 菌体细胞 表面的结构蛋白, 由 293 个氨基酸残基组成, 成熟的PF分 子量为 $30.1 \mathrm{kD}[11]$, 其羧基末端具有一个赖氨酸残基。 研究表明PF参与了 NTHi逃避宿主补体免疫的过程, 促进了 NTHi 与Plg、层粘连蛋白（Laminin, Ln) 、玻粘连蛋白 (Vitronectin，Vn）及支气管上皮细胞的结合 [12]。

Lp (a) 是一种特殊的血浆脂蛋白, 由Apo (a) 和LDL通过 二硫键连接而成。Apo (a) 中的K结构域与 Plg的K结构域高 度同源。Apo (a) 中也具有很强的LBS, 能够结合赖氨酸及 其类似物。韩润林教授提出的 $L p(a)$ 抗感染假说指出: Lp (a) 可能竞争性的抑制细菌与Plg的结合, 从而抑制病原菌在 宿主中的扩散 [13]。

本研究将从NTHi 基因组中扩增出的 $h p f$ 片段连入 pGEX-6p-1表达载体并将其转入 E. coli BL21中, 诱导表 达出 $r P F$ 及缺失羧基末端赖氨酸残基的 $r P F \Delta \mathrm{k}$ 。在此基础 上对 $r P F$ 与 $P 1 g 、 \operatorname{Lp}(a)$ 的相互作用进行初步研究, 为进一 步证实Lp (a) 抗感染机制提供了依据。

\section{2. 材料与方法}

\section{1. 菌株和试剂}

NTHi ATCC49247购自卫生部临床检验中心; 表达载体 pGEX-6p-1购自上海林渊生物科技有限公司; 限制性内切
酶BamH I、Not I、DL 5000 DNA Marker均购自宝生物工 程 (大连) 有限公司; T4 DNA连接酶购自Promega公司; 细菌基因组DNA提取试剂盒购自北京天根生物科技有限公 司; Lp (a)、羊抗人 Apo (a) 多抗购自购自Biomedical Technologies Inc公司; EACA、羊抗人LDL多抗购自Sigma 公司; Plg、鼠抗人纤溶酶原单抗、驴抗山羊 $I g G-H R P$ 购自 R\&D Systems公司; 山羊抗鼠 IgG-HRP购自北京博奥森生 物技术有限公司; Pierce ${ }^{\circledR} G 1$ tathione Agarose、 GST-probe-HRP、Pierce BCA蛋白测定试剂盒购自 Thermo 公司; 大肠杆菌 E. coli BL21、Prescission 酶、引物 合成均购自生工生物工程（上海）股份有限公司。所用引 物见下表:

表1 引物列表。

\begin{tabular}{lll}
\hline $\begin{array}{l}\text { Target } \\
\text { gene }\end{array}$ & Primer: Secquence $\left(5^{\prime}-\mathbf{- 3},\right)$ & $\begin{array}{l}\text { PCR } \\
\text { product } / \text { bp }\end{array}$ \\
\hline & F : CGCGGATCCAAATTTAAAGTCGTAACCACT & \\
$H p f$ & R1: ATTTGCGGCCGCTCATTTTCCGAATCCTTTAAC & 837 \\
$h p f \Delta \mathrm{k}$ & R2: ATTTGCGGCCGCTCATCCGAATCCTTTAACAATGG & 834 \\
\hline
\end{tabular}

注：上游引物为共用引物; 下划线处为 BamH I及 $N o t$ I酶识别位点

\section{2. 目的基因的克隆及连接转化}

按照细菌基因组DNA提取试剂盒操作说明进行NTHi基 因组DNA的提取。以提取的基因组DNA为模板, 利用引物F、 $\mathrm{R} 1$ 扩增 $h p f$ 基因; 利用引物 $\mathrm{F} 、 \mathrm{R} 2$ 扩增 $h p f \Delta \mathrm{k}$ 基因。PCR扩 增条件为: $94^{\circ} \mathrm{C}$ 预变性 $3 \mathrm{~min} ; 94^{\circ} \mathrm{C}$ 变性 $30 \mathrm{~s} ; 55^{\circ} \mathrm{C}$ 退火 $30 \mathrm{~s}$; $72^{\circ} \mathrm{C}$ 延伸 $1 \mathrm{~min}, 30$ 个循环; $72^{\circ} \mathrm{C}$ 总延伸 $2 \mathrm{~min}$ 。

将PCR扩增得到的产物纯化后进行双酶切。将酶切产 物进行纯化, 连接到 $\mathrm{pGEX}-6 \mathrm{p}-1$ 表达载体上, 构建 pGEX-6p-1-hpf、pGEX-6p-1-hpf $\Delta \mathrm{k}$ 重组表达载体, 并转 入感受态 $E$. coli BL21中。

利用通用引物进行菌落PCR鉴定，并将阳性菌送交生 工生物工程（上海）股份有限公司测序，对测序结果正确 的菌株进行冻存保菌。

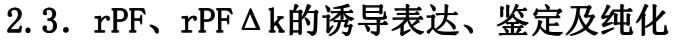

\section{3. 1. $\mathrm{rPF} 、 \mathrm{rPF} \Delta \mathrm{k}$ 的诱导表达}

取含有pGEX-6p-1-hpf、pGEX-6p-1-hpf $\Delta \mathrm{k}$ 重组表达 载体的工程菌 $E$. coli BL21接种于 $20 \mathrm{~mL} \mathrm{LB} / \mathrm{Amp}$ (Amp含 量为 $100 \mu \mathrm{g} / \mathrm{mL}$ ) 液体培养基中, 于恒温摇床上 $37^{\circ} \mathrm{C}, 200$ $\mathrm{rpm}$ 振荡培养 $16 \mathrm{~h}$; 转接至 $400 \mathrm{~mL}$ 培养基中, $37^{\circ} \mathrm{C} 、 200 \mathrm{rpm}$ 振荡培养至 $0.6 \leqslant 0 D_{600 \mathrm{~nm}} \leqslant 0.8$; 向培养基中加入 IPTG（终 浓度为 $1 \mathrm{mmol} / \mathrm{L}), 30^{\circ} \mathrm{C} 、 300 \mathrm{rpm}$, 恒温摇床振荡 $3 \mathrm{~h}$ 进行诱导培养。

将诱导后的菌液离心收集沉淀并用PBS重悬, 置于 $-80^{\circ} \mathrm{C}$ 冰箱冻融 3 次; 将冻融的菌悬液融化后加入 PMSF ( 1 $\mathrm{mmol} / \mathrm{L})$, 溶菌酶 $(0.2 \mathrm{mg} / \mathrm{mL})$, DNA酶 $(20 \mu \mathrm{g} / \mathrm{mL})$, 震荡30 $\mathrm{min} ; 4^{\circ} \mathrm{C} 、 10000 \mathrm{rpm}$ 离心 $20 \mathrm{~min}$, 收集破碎后 的菌沉淀和菌上清。 


\subsubsection{SDS-PAGE鉴定}

取样制备诱导前、诱导后及诱导破碎后菌上清、菌沉 淀的电泳样品并进行SDS-PAGE电泳（4\%浓缩胶，12\%分离 胶）。电泳完成后，经考马斯亮兰染色、脱色、照相。

\subsection{3. 重组蛋白 $r P F 、 r P F \Delta \mathrm{k}$ 的纯化}

将得到的裂解后的菌上清用Wash buffer稀释后进行 过柱：上清稀释液穿流3次; 然后用Wash buffer对结合在 柱填料上的蛋白进行清洗，除去杂蛋白; 最后加入 2 倍体 积的Elution buffer，封住出口，室温睬育20 min; 然后 收集洗脱液并用 6 个柱体积的Elution buffer洗脱结合在 柱子上的蛋白，收集洗脱液。得到的洗脱液过夜透析去除 杂质。

将透析得到的rPF、rPF $\Delta \mathrm{k}$ 用PEG 20000 进行浓缩后 测定蛋白含量, 并分装保存。

\section{4. $\mathrm{rPF} 、 \mathrm{rPF} \Delta \mathrm{k}$ 标签 (GST) 的切除}

按照 $2 \mathrm{U} / 100 \mu \mathrm{g}$ 的量向已知含量的 $r P F$ 和 $r P F \Delta k$ 蛋白液 中加入Prescission酶, $4{ }^{\circ} \mathrm{C}$ 捊育 $24 \mathrm{~h}$; 然后将蛋白液加 入已经用Cut buffer平衡的柱子中, 封住出口在室温下捊 育 $30 \mathrm{~min}$; 打开出口收集流出液, 并用 4 个柱体积的Cut buffer洗脱目的蛋白进行收集。将切除标签后的蛋白过夜 透析、浓缩并测定含量, 分装保存。

\section{5. 标签切除效果的Western-blot检测}

$r P F 、 r P F-G S T 、 r P F \Delta k 、 r P F \Delta k-G S T$ 原液各取 $10 \mu \mathrm{L}$ 用PBS进行如下稀释: $r P F 、 r P F \Delta k$ 为 $20 \mu \mathrm{g} / \mathrm{mL}$; $r P F-G S T$ 、 $\mathrm{rPF} \Delta \mathrm{k}-\mathrm{GST}$ 为 $37.3 \mu \mathrm{g} / \mathrm{mL}$ (同摩尔数)。取上述各浓度 的蛋白液作样后进行SDS-PAGE电泳（浓缩胶 $4 \%$, 分离胶 $10 \%$ ）。电泳后利用半干转法将蛋白转移到 NC膜上, 将NC 膜放入 $5 \%$ 的脱脂乳-TBST中室温封闭 $1.5 \mathrm{~h}$; 加入用 $2 \%$ 脱脂 乳-TBST稀释 (1:2500) 的GST标签抗体, 室温捊育1.5 h; 加入发光试剂, 然后进行成像操作。

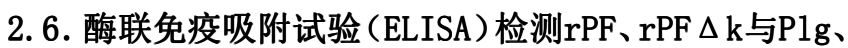 $\operatorname{Lp}(\mathrm{a})$ 的结合}

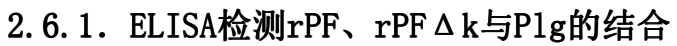

将 $r P F 、 r P F \Delta k$ 稀释为 $20 \mu \mathrm{g} / \mathrm{mL}$, 按照 $100 \mu \mathrm{L} /$ 孔将 $r P F 、 r P F \Delta \mathrm{k}$ 包被在酶标板中室温蜉育 $1.5 \mathrm{~h}$; TBST洗板 3 次, $5 \mathrm{~min} /$ 次; 向酶标板各孔中加入 $1 \% B S A-T B S T$ 室温封闭 $1.5 \mathrm{~h}$; 对应各孔加入不同浓度的P $1 \mathrm{~g}(10 、 50 、 100 \mathrm{ng} / 100$ $\mu \mathrm{L}$ ) $100 \mu \mathrm{L} /$ 孔, 室温孵育 $1.5 \mathrm{~h}$; 将鼠抗人P1g单抗 (TBST $1: 2000$ 稀释）加入对应各孔，100 $\mu \mathrm{L} /$ 孔, 室温孵育 1.5 $\mathrm{h}$; 将山羊抗鼠 IgG-HRP（TBST 1:3000稀释）加入对应孔 中, $100 \mu \mathrm{L} /$ 孔, 室温捊育 $1.5 \mathrm{~h}$; 加入四甲基联苯胺 (TMB) 显色液, 室温作用 $5 \mathrm{~min}$; 加入终止液, 于酶标仪上测定 $0 \mathrm{D}_{450 \mathrm{~nm}} \mathrm{o}$

\subsection{ELISA检测 $r P F 、 r P F \Delta k$ 与 $L p(a)$ 的结合}

实验方法如1.6. 所述。 $r P F 、 r P F \Delta$ 浓度为 $20 \mu \mathrm{g} / \mathrm{mL}$, $\mathrm{Lp}(\mathrm{a})$ 浓度梯度为 $10 、 50 、 100 \mathrm{ng} / 100 \mu \mathrm{L}$ 。使用一抗山
羊抗人Apo (a) 多抗（TBST 1:4 000稀释）；二抗驴抗山羊 IgG-HRP（TBST 1:1 000稀释）。

\subsection{3. 亲和色谱层析检测 $r P F 、 r P F \Delta k$ 与 $L p(a)$ 的结合}

在三根已加入GST填料的层析柱中分别加入 $50 \mu \mathrm{g}$ 的 $r P F 、 r P F \Delta \mathrm{k}$ 以及相应的PBS。然后向每个层析柱中加入 1 $\mathrm{mL}$ 血浆, 作用 $30 \mathrm{~min}$ 后用Wash buffer洗去未结合的杂蛋 白。向三根层析柱中加入 $10 \mathrm{U}$ 的Prescission酶, $4{ }^{\circ} \mathrm{C}$ 䁔育 $24 \mathrm{~h}$, 用Cut buffer洗脱目的蛋白。

将上述得到的洗脱液处理后作样进行SDS-PAGE，而后 用湿转电转仪将蛋白转移到 NC膜上, 剩余操作同 1.5 。检 测 Lp (a) 使用的一抗为羊抗人 Apo (a)，二抗为驴抗羊 IgG-HRP; 检测 $r \mathrm{PF} / \mathrm{rPF} \Delta \mathrm{k}$ 使用的是GST-probe-HRP抗体。

\section{7. ELISA检测EACA对 $r P F$ 与Plg、 Lp (a) 结合的抑制}

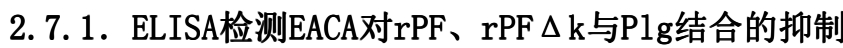

实验步骤及抗体使用与 1.6 .1 操作一致。 $\mathrm{P} 1 \mathrm{~g}$ 的浓度为 $100 \mathrm{ng} / 100 \mu \mathrm{L}, \mathrm{EACA}$ 终浓度为0、0.2、2. $0 \mathrm{mmol} / \mathrm{L}$ 。剩 余步骤同1.6.1

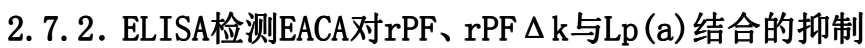

实验步骤及抗体使用与 1.6 .2 一致。 $\mathrm{Lp}$ (a) 的浓度为 $100 \mathrm{ng} / 100 \mu \mathrm{L}, \mathrm{EACA}$ 终浓度依次为 $0 、 0.2 、 2.0 \mathrm{mmol} / \mathrm{L}$ 。 剩余步骤同1.6.2

\section{8. ELISA检测 $L p(a)$ 对 $r P F$ 与Plg结合的抑制}

实验步骤与1.6.1一致。 $\mathrm{P} 1 \mathrm{~g}$ 的浓度为 $100 \mathrm{ng} / 100 \mu \mathrm{L}$, $\mathrm{Lp}$ (a) 终浓度为 $0 、 50 、 100 、 200 \mathrm{ng} / 100 \mu \mathrm{L}$ 。一抗、二 抗的使用及稀释比例均与 1.6.1所述一致。

注: ELISA检测结果采用SAS软件进行统计分析, 并进 行t检验。

\section{3. 结果}

\section{1. 标签切除效果的Western-blot检测}

由于标签蛋白本身分子量过大 $(26 \mathrm{kD})$, 为了不影 响后续对目的蛋白的研究, 使用Prescission酶对标签蛋 白进行剪切。利用GST标签抗体对重组蛋白标签的切除效 果进行检测, rPF-GST、rPF $\Delta \mathrm{k}-\mathrm{GST}$ 大小为 $56 \mathrm{kD}$, 切除标 签后的 $r P F 、 r P F \Delta k$ 大小为 $30 \mathrm{kD}$ 。 $r P F-G S T 、 r P F \Delta k-G S T$ 在预期位置均有明显条带, 而rPF、rPF $\Delta k$ 预期大小处完 全没有条带（见图1）。说明利用Prescission酶对重组蛋 白实施的标签切除很干净。

\section{2. ELISA检测 $r P F 、 r P F \Delta k$ 与Plg、 $L p(a)$ 的结合}

\section{2. 1. ELISA检测 $r P F 、 r P F \Delta k$ 与Plg的结合}

ELISA检测纯化后的rPF、rPF $\Delta \mathrm{k}$ 与Plg之间的相互作 用, 结果 (见图 $2 A$ ) 表明: $r P F 、 r P F \Delta k$ 与 Plg 的结合均具 有一定的浓度依赖性, 随着P1g浓度的升高结合值也在增 大；同P1g浓度下，相比于rPF $\Delta \mathrm{k}, \mathrm{rPF}$ 与Plg的结合能力 显著增强 $(p<0.01)$ 。 


\section{2. 2. ELISA检测 $r P F 、 r P F \Delta \mathrm{k}$ 与 $L p$ (a) 的结合}

$r P F 、 r P F \Delta \mathrm{k}$ 与 $L p(a)$ 的ELISA检测结果（见图2B）表

明: $r P F 、 r P F \Delta k$ 与 $L p(a)$ 的结合具有浓度依赖性, 在 10-100 $n g / 100 \mu \mathrm{L}$ 浓度范围内 $r P F 、 r P F \Delta \mathrm{k}$ 与 Lp (a) 的结合值随 $\mathrm{Lp}(\mathrm{a})$ 浓度的增高而增高。相比于rPF $\Delta \mathrm{k}, \operatorname{rPF}$ 与 Lp (a) 的 结合值在各浓度水平下均明显升高 $(p<0.01)$ 。

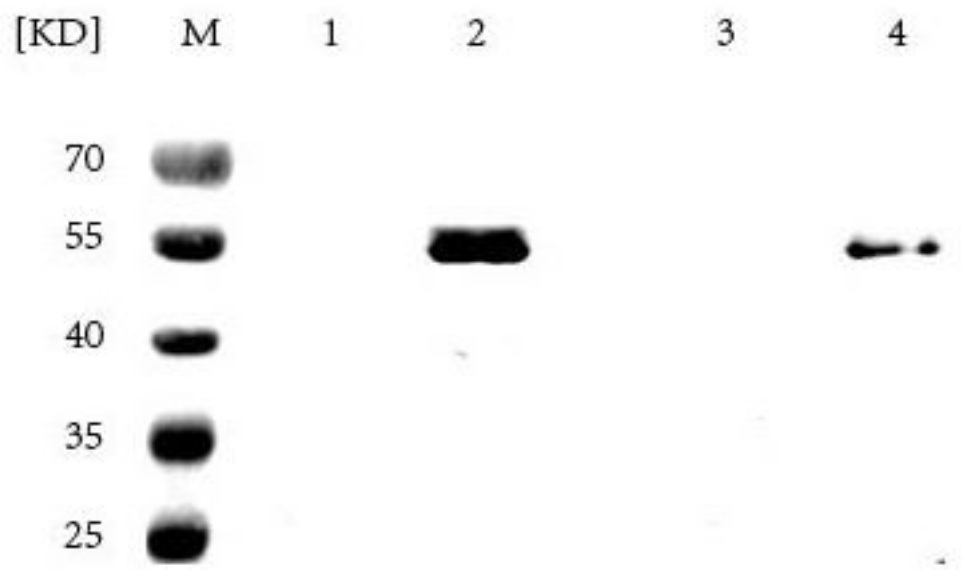

M：蛋白质分子质量标准; 1 ：切除标签的rPF；2：未切除标签的 $\mathrm{PPF} ; 3$ ：切除标签的 $\mathrm{PPF} \Delta \mathrm{k} ; 4$ ：未切除标签的rPF $\Delta \mathrm{k}$

图1 重组蛋白标签切除的Western-blot分析。
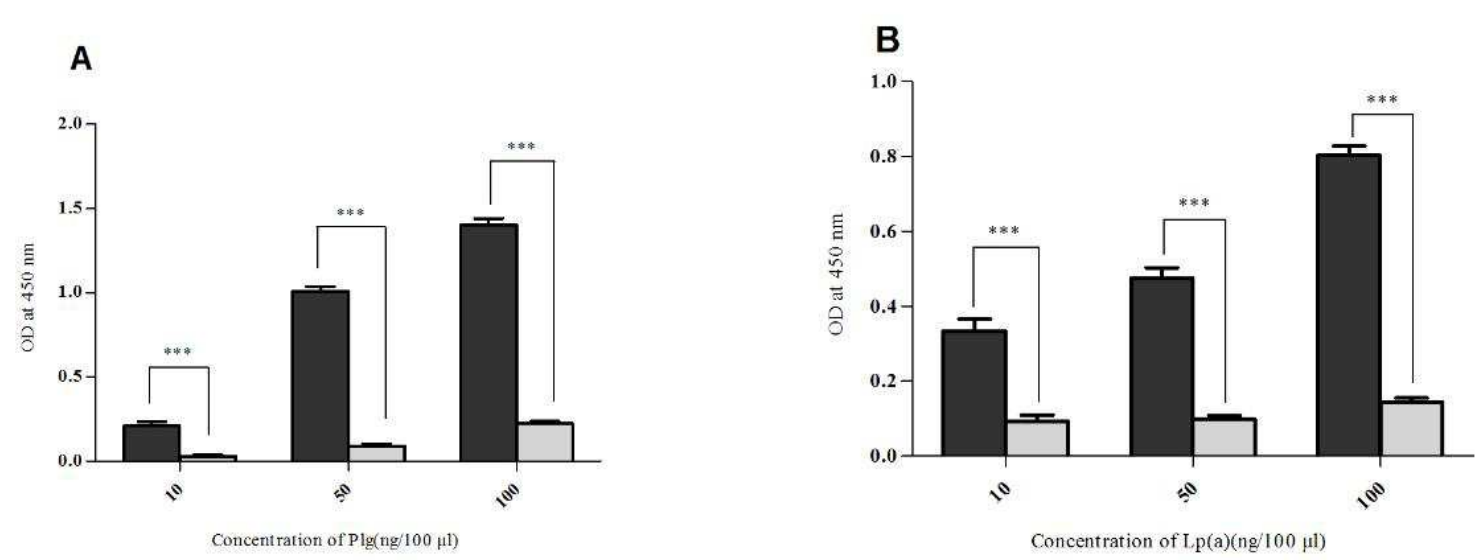

A：rPF、rPFAk 与PIg的结合 B：rPF、rPFAk与Lp(a)的结合 ***: $(\mathbf{p}<0.01)$

图2 ELISA检测 $r P F 、 r P F \Delta \mathrm{k}$ 与 Plg、 Lp (a) 的结合。

\subsection{3. 亲和色谱层析检测 $r P F 、 r P F \Delta k$ 与 $L p(a)$ 的结合}

将亲和色谱层析得到的洗脱液进行Western检测, 由 结果（见图3）可知: rPF、rPF $\Delta \mathrm{k}$ 与 Lp (a) 均结合, 但在
亮度上具有明显的差异。这表明 $r P F$ 羧基末端的赖氨酸残 基是主要的结合位点。

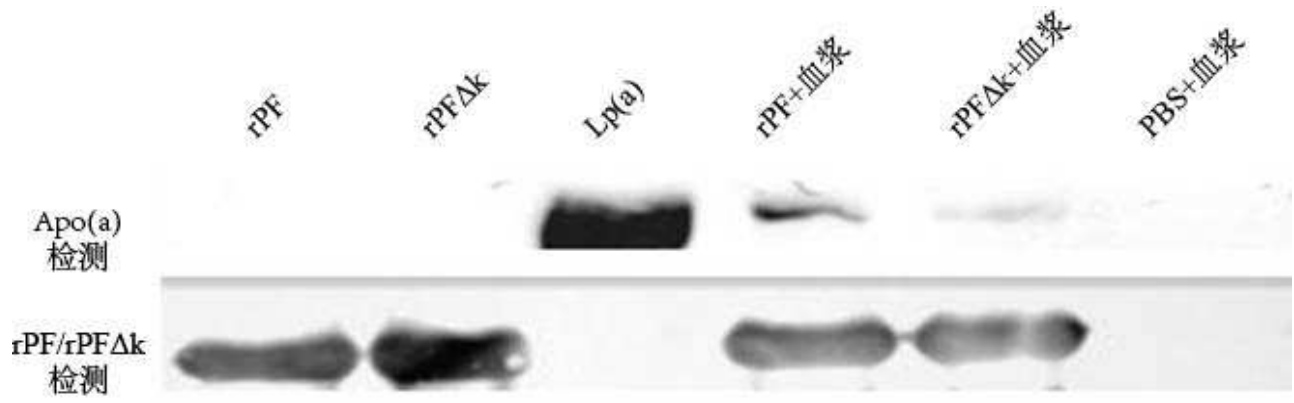

图3 Western blot 检测 $r P F 、 r P F \Delta k$ 与 Lp (a) 的结合。 


\section{3. ELISA检测EACA对 $r P F 、 r P F \Delta k$ 与Plg、 $L p(a)$ 结合的 抑制}

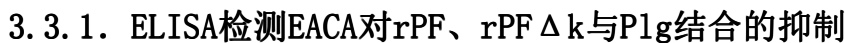

ELISA检测EACA对 $r P F 、 r P F \Delta k$ 与Plg 结合的抑制结果 （见图4A）表明：随着EACA浓度的增高, rPF与Plg的结合 值在不断降低，当EACA浓度为 $2.0 \mathrm{mmol} / \mathrm{L}$ 时，其抑制作用 较浓度为 $0 \mathrm{mmol} / \mathrm{L}$ 时具有显著性差异 $(p<0.01)$ 。

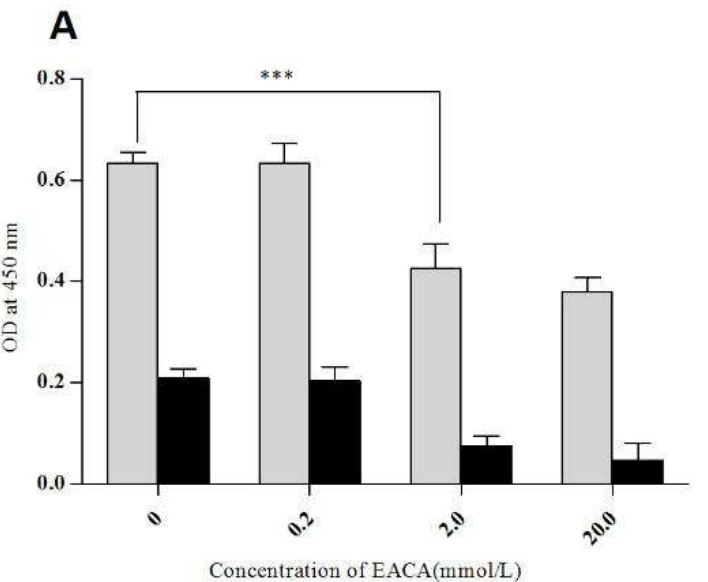

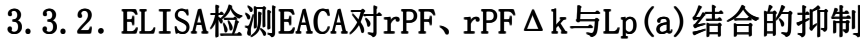

ELISA检测EACA对 $r P F 、 r P F \Delta k$ 与Lp (a) 结合的抑制, 结果 (见图4B) 表明: 随着EACA浓度的增高, rPF与Lp (a) 的结合值在不断降低, 当EACA浓度为 $0.2 \mathrm{mmol} / \mathrm{L}$ 时, 其抑 制作用较EACA浓度为 $0 \mathrm{mmol} / \mathrm{L}$ 时具有显著性差异 $(p<$ 0.01 )。

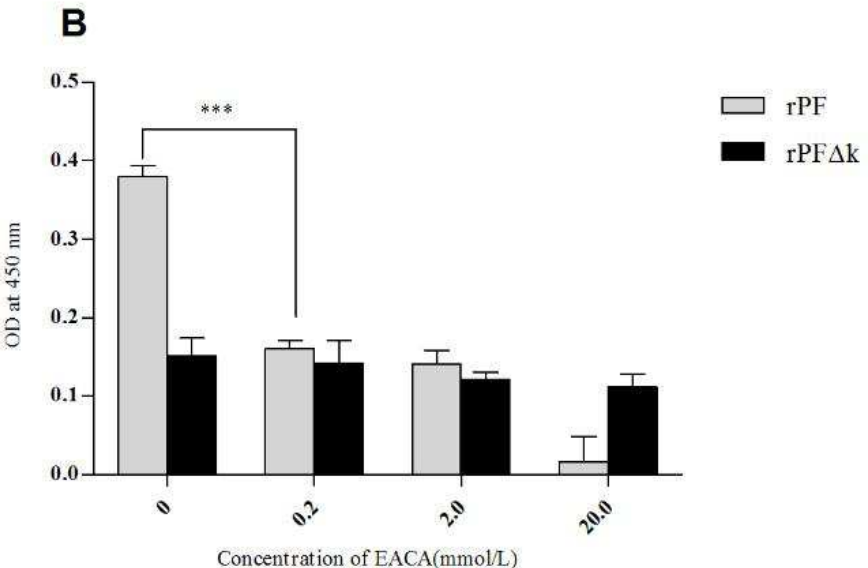

A： EACA对 rPF, rPFAk 与 Plg结合的抑制 B： EACA对 rPF, rPFAk 与 Lp(a)结合的抑制 ***: $p<0.01$

图4 EACA对 $r P F, r P F \Delta k$ 与Plg、Lp (a) 结合的抑制。

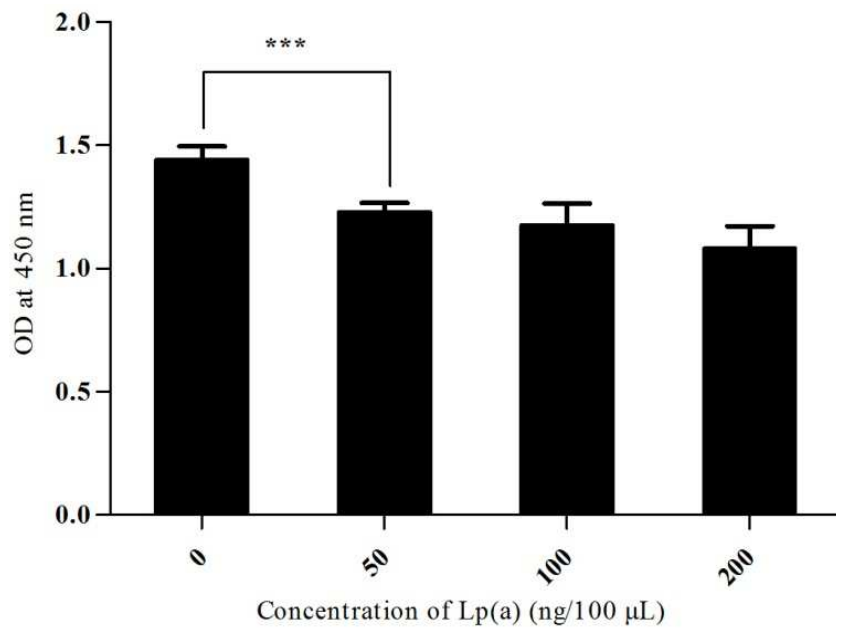

图5 Lp (a) 对 rPF与P1g结合的抑制 $* * *: p<0.01$ 。

\section{4. ELISA检测 $L p(a)$ 对 $r P F$ 与Plg结合的抑制}

ELISA检测Lp (a) 对 $r P F$ 与Plg结合的抑制作用试验结 果（见图5）表明: Lp (a) 对 rPF与Plg结合的抑制具有一定 的浓度依赖性，当Lp (a) 浓度达到50 ng/100 $\mu$ L时，抑制 作用显著增强 $(p<0.01)$ 。

\section{4. 讨论}

PF是一种羧基末端含有赖氨酸残基的菌体表面蛋白。 $P 1 g 、 L p(a)$ 均具有 LBS, 能够与赖氨酸及其类似物相结合
[14], 由本实验的研究结果可知, rPF能够与Plg、Lp (a) 结合, 并且EACA能够抑制这种结合作用。rPF $\Delta \mathrm{k}$ 与 P $1 g$ 、 $\mathrm{Lp}(\mathrm{a})$ 的结合值明显降低, 说明 $r P F$ 与 P lg、 Lp (a) 的结合作 用主要是通过 $r P F$ 羧基末端的赖氨酸残基与 P $1 g 、 L p(a)$ 上 的LBS特异性结合而形成的。

此外, 本研究发现 $r P F \Delta \mathrm{k}$ 与 Plg、Lp (a) 仍然具有结合 值。这说明 $r P F$ 内部的其他赖氨酸残基也参与了与 $\mathrm{P} 1 \mathrm{~g}$ 、 $\mathrm{Lp}$ (a) 的结合作用，但发挥主要作用的是羧基末端的赖氨 酸残基，这一点通过ELISA及亲和色谱层析等检测方法均 得到了证实。

$L p(a)$ 抑制 $r P F$ 与 $P 1 g$ 结合作用的研究表明, $L p(a)$ 能够 竞争性的抑制 Plg 与 $r P F$ 的结合，当 $\mathrm{Lp}$ (a) 浓度达到 50 $n g / 100 \mu \mathrm{L}$ 时，就能够产生显著的抑制作用。

人们对于 $L p(a)$ 与肾脏疾病及动脉粥样硬化等疾病关 系的研究有所突破 [15], 但 $L p(a)$ 具体的生理作用仍不明 确。通过本实验的研究可知 $L p(a)$ 能够抑制细菌表面PF与 $\mathrm{P} 1 \mathrm{~g}$ 的结合, 由此推断 $\mathrm{Lp}(\mathrm{a})$ 能够阻断细菌利用 $\mathrm{P} 1 \mathrm{~g}$ 侵袭宿 主的途径, 在抵抗NTHi感染方面具有重要作用。

\section{参考文献}

[1] Watt JP, Wolfson LJ, Henkle E, et al. Burden of disease caused by Haemophilus influenzae type b in children younger than 5 years: global estimates $[J]$. Lancet, 2009, 374(9693); $903-911$. 
[2] Morris SK. Moss WJ, Halesa N, et al. Haemophilus influenzae type $\mathrm{b}$ conjugate vaccine use and effectiveness $[J]$. Lancet Infectious Diseases, 2008, $8(7) ; 435-443$.

[3] Erwin AL, SmithAL. Nontypeable Haemophilus influenzae: understanding virulence and commensal behavior[J]. Trends Microbiol, 2007, 15(8); $355-362$.

[4] Murphy TF, Faden H, Bakaletz L0, et al. Nontypeable Haemophilus influenzae as a pathogen in children[J]. Pediatric Infectious Diseases, 2009, 28(1); 43 - 48.

[5] Turk DC. The pathogenicity of Haemophilus influenzae[J]. J Med Microbiol, 1984, 18:1-16.

[6] Lahteenmaki K, Kuusela P, Korhonen TK. Bacterial plasminogen activators and receptor $[J]$. FEMS Microbiology Reviews, 2001, $25(5): 531-552$.

[7] Redlitz A, Plow EF. Receptors for plasminogen and t-PA: an update[J]. Baillieres Clinical Haematology, 1995, $8(2): 313-327$.

[8] Barthel D, Singh B, Riesbeck K, et al. Haemophilus influenzae uses the surface protein $\mathrm{E}$ to acquire human plasminogen and to evade innate immunity[J]. Immunology, 2012, 188(1) ; 379-385.

[9] Sjostrom I, Grondahl H, Falk G, et al. Purification and characterisation of a plasminogen-binding protein from Haemophilus influenzae. Sequence determination reveals identity with aspartase[J]. Biochimica et Biophysica Acta, 1997, 1324(2) ; 182 190.

[10] Su YC, Mukher jee 0, Singh B, et al. Haemophilus influenza P4 interacts with extracellular matrix proteins promoting adhesion and serum resistance [Z]. http://jid. oxford journals. org, 2015-8-7.

[11] Jalalvand F, Su YC, Mörgelin M, et al. Haemophilus influenzae protein $\mathrm{F}$ mediates binding to laminin and human pulmonary epithelial cells[J]. Infectious Diseases, 2013, 207 (5); 803-813.

[12] Su YC, Jalalvand F, Mörgelin M, et al. Haemophilus influenza acquires vitronectin via the ubiquitous protein $F$ to subvert host innate immunity[J]. Molecular Microbiology, 2013, 87 (6) ; 1245 - 1266.

[13] Han RL. Plasma lipoproteins are important components of the immune system[J]. Microbiol Immunol. 2010, 54 (4) ; 246-253.

[14] Boeryd B. Action of heparin and plasminogen inhibitor (EACA) on metastatic tumour spread in an isologous system[J]. Acta pathologica et microbiologica Scandinavica. 1965, 65 (3) :395-404.

[15] Mclean JW, Tomlinson JE, Kuang WJ, et al. cDNA secquence of human apolipoprotein (a) is homologous to plasminogen[J]. Nature, 1987, 330 (6144) :132-137. 